\title{
Injectable Click-Crosslinked Hyaluronic Acid Depot to Prolong Therapeutic Activity in Articular Joints Affected by Rheumatoid Arthritis
}

Jiyoung Seo ${ }^{1}$, Seung Hun Park ${ }^{1}$, Min Ju Kim${ }^{1}$, Hyeon Jin $\mathrm{Ju}^{1}$, Xiang Yun Yin ${ }^{2}$, Byoung Hyun Min ${ }^{1,2}$, Moon Suk $\mathrm{Kim}^{1, *}$

${ }^{1}$ Department of Molecular Science and Technology, Ajou University, Suwon 16499, Korea

${ }^{2}$ Department of Orthopedic Surgery, School of Medicine, Ajou University, Suwon 16499, Korea

CORRESPONDING AUTHOR: moonskim@ajou.ac.kr, Tel: 82-31-219-2608, Fax: 82-31-219-3931 


\section{Experimental Section.}

\section{Characterization of $\mathrm{HA}$ and $\mathrm{Cx}-\mathrm{HA}$}

Employing a rheometer (MCR 102, Anton Paar, Ostfildern, Germany) with a Peltier temperaturecontrolled bottom plate and a $25.0 \mathrm{~mm}$ stainless-steel parallel-plate measuring system, the HA and CxHA solutions' rheological properties were investigated. The gap length and temperature were $0.3 \mathrm{~mm}$ and $25^{\circ} \mathrm{C}$, respectively. The time sweep was $0-100 \mathrm{~s}$, and it had an oscillating frequency of $1 \mathrm{~Hz}$ and $2 \%$ amplitude $\gamma$. Frequency was measured at an oscillating frequency of $0.1-10 \mathrm{~Hz}$ and $2 \%$ amplitude $\gamma$. The strain sweep was made at an oscillating strain of $0.1-1000 \%$, with an oscillating frequency of $1 \mathrm{~Hz}$. The storage modulus $\left(G^{\prime}\right)$, loss modulus $\left(G^{\prime \prime}\right)$, viscosity $(\eta)$, and phase angle $(\tan \delta)$ were calculated by the software included with the instrument.

HA and Cx-HA specimens were prepared for scanning electron microscopy (SEM) by coating lyophilized HA and Cx-HA with a conductive layer of gold using a plasma-sputtering apparatus (Emitech, K575, Kent, UK). SEM images were captured by field-emission SEM (FE-SEM; JSM-6700F, JEOL, Tokyo, Japan).

\section{Determination of degradation and swelling properties of $\mathrm{HA}$ and $\mathrm{Cx}-\mathrm{HA}$}

First, $200 \mu \mathrm{L}$ of the prepared $\mathrm{Cx}-\mathrm{HA}$ and HA (4mg Cx-HA or HA) were aliquoted into 20mL vials, $10 \mathrm{~mL}$ of PBS was added, and the mixtures were incubated at $37^{\circ} \mathrm{C}$. At predetermined time points, the PBS not absorbed by the Cx-HA was removed from the vials. The PBS left on the surface of the vials was removed using clean wipes (Yuhan-Kimberly, Seoul, Korea). Vials were then weighed to calculate the weight of wet $\mathrm{Cx}-\mathrm{HA}$. Each $\mathrm{Cx}-\mathrm{HA}$ hydrogel was then lyophilized in a freeze dryer until the residue reached a constant weight, and the vial was then weighed.

The following equation was used to define the swelling ratio: Swelling ratio $(\%)=[$ (weight of wet 
Cx-HA - weight of dry Cx-HA) $\div$ (weight of dry Cx-HA)] $\times 100$.

The degradation ratio was defined as follows: Degradation ratio $(\%)=[($ weight of dry $\mathrm{Cx}-\mathrm{HA}) \div$ (weight of initial Cx-HA)] $\times 100$.

\section{In vitro MTX release}

One milliliter of MTX-HA (37.5 $\mu \mathrm{g}$ of MTX per $20 \mathrm{mg}$ of HA) was loaded into a dialysis tube with a molecular weight cutoff of $3.5 \mathrm{kDa}$. A solution of $500 \mu \mathrm{L}$ of MTX-TET-HA (18.75 $\mu \mathrm{g}$ of MTX per $10 \mathrm{mg}$ of TET-HA) and $0.5 \mathrm{~mL}$ of MTX-TCO-HA (18.75 $\mu \mathrm{g}$ of MTX per 10mg of TCO-HA) was added to the dialysis tube to prepare MTX-Cx-HA. An additional $3 \mathrm{~mL}$ of PBS was added to the $20 \mathrm{~mL}$ vials of the MTX-HA and MTX-Cx-HA depots, and the MTX-HA and MTX-Cx-HA depots were incubated at $100 \mathrm{rpm}$ at $37^{\circ} \mathrm{C}$. For each experiment, $1 \mathrm{~mL}$ of a solution was extracted from the $20 \mathrm{~mL}$ vial at predetermined time points, and $1 \mathrm{~mL}$ of fresh PBS was immediately added to the $20 \mathrm{~mL}$ vial. A high-

performance liquid chromatography system (Agilent 1200 series, Agilent Technologies, Waldbronn, Germany) at a wavelength of $360 \mathrm{~nm}$ and a column flow rate of $1 \mathrm{~mL} / \mathrm{min}$ was used to detect the MTX released from MTX-HA and MTX-Cx-HA. For the mobile phase, a mixture of $0.1 \mathrm{M}$ sodium phosphate and acetonitrile $(90: 10, \mathrm{v} / \mathrm{v})$ was employed. All samples were examined in triplicate. Using standard calibration curves created with known concentrations of MTX, the amounts of MTX released from MTX-HA and MTX-CX-HA were determined.

\section{Inflammation assays using RAW 264.7 and synovial cells}

RAW 264.7 cells $\left(10^{4}\right.$ per well $)$ and SW 982 cells $\left(3 \times 10^{4}\right.$ per well $)$ as synovial cells were prepared by a method similar to the one described in the in previous work in references 18 and 19. One hundred microliters of HA $(20 \mathrm{mg} / \mathrm{mL})$, Cx-HA prepared by mixing TET-HA $(10 \mathrm{mg} / \mathrm{mL})$ and TCO-HA $(10 \mathrm{mg} / \mathrm{mL})$, or MTX $(37.5 \mu \mathrm{g} / \mathrm{mL}$ in PBS) was added to the upper chambers $(0.4 \mu \mathrm{m}$ membrane) of a $24-$ 
well Transwell plate. One hundred microliters of MTX-HA or MTX-Cx-HA (MTX:HA or MTX:CxHA ratio $=37.5 \mu \mathrm{g} / \mathrm{mL}$ to $20 \mathrm{mg} / \mathrm{mL}$ ) was also added to the upper chambers.

Media in the bottom chambers was changed by refreshing each culture medium every day for 7 days. On days 1, 4, and 7, the 24-well Transwell plate's upper chambers were removed. The proliferation of RAW 264.7 or synovial cells was determined using the alamarBlue assay (Life Technologies, Camarillo, CA, USA). A $10 \%$ alamarBlue solution in $1 \mathrm{~mL}$ DMEM was added to the cell cultures in the bottom chambers of the 24-well Transwell plates and incubated for $4 \mathrm{~h}$. Aliquots of the alamarBlue solution from the bottom chambers of the 24-well Transwell plates were transferred to a 96-well plate, and their absorbance was measured on a Cytation3 imaging reader (BioTek, VT, USA) at wavelengths of 570 and $600 \mathrm{~nm}$. All of the experiments were independently conducted in triplicate, and the results are presented as mean \pm standard deviation (SD).

To determine the concentration of inflammatory cytokine tumor necrosis factor alpha (TNF- $\alpha$ ), RAW 264.7 cells treated with HA alone, Cx-HA alone, MTX alone, MTX-HA, or MTX-Cx-HA were stimulated with $1 \mathrm{mg} / \mathrm{mL}$ lipopolysaccharides (Escherichia coli 055:B5; Sigma) for $24 \mathrm{~h}$. The TNF- $\alpha$ concentration in the culture supernatant of the stimulated cells was then measured using mouse TNF- $\alpha$ ELISA Kits (R\&D Systems, MN, USA) at days 1, 4, and 7. All of the experiments were conducted at least in triplicate, and the results are presented as mean $\pm \mathrm{SD}$.

\section{NIR fluorescence imaging of NIR-HA and NIR-Cx-HA in vivo}

Twenty milligrams of NIR-HA was dissolved in $1.0 \mathrm{~mL}$ of PBS and loaded into a single-barrel syringe. Ten mg of NIR-TET-HA and 10mg of NIR-TCO-HA were separately solubilized in $0.5 \mathrm{~mL}$ of PBS, and each solution was loaded into one compartment of a dual-barrel syringe. One hundred microliters of each formulation was injected into the articular knee joints of the RA rats using $26 \mathrm{G}$ syringes. At various time points, fluorescent NIR images were acquired at a wavelength of $780 \mathrm{~nm}$ using 
a FOBI imaging instrument (NeoScience, Suwon, Korea). NIR pictures were taken at an exposure time of $1000 \mathrm{~s}$ with a dichroic cube filter $\left(\mathrm{MgF}_{2}\right.$ fused silica filter $)$.

\section{Micro-computed tomography (CT) images of $R A$ rats}

The therapeutic efficiency of each formulation in the treated RA rats was analyzed using micro-CT with a Skyscan 1076 (Skyscan; Kontich, Belgium) instrument (18.22 $\mu \mathrm{m}$ pixel resolution, 340ms exposure time, $59 \mathrm{kV}$ energy source, $153 \mathrm{~mA}$ current). The measurement was performed by a method similar to the one described in previous work in reference 17 . The volume ratio of new bone (BV) to tissue (TV) [BV/TV] was determined from the CT images using the MIMICS 16.0 software.

\section{Histology}

The treated RA and normal rats were euthanized after 1, 3, and 6 weeks, and the articular knee joints were excised from individual rats ( $n=3$ for each time point). The articular knee joints were fixed with $10 \%$ formalin for 4 days and decalcified using $6 \%$ nitric acid for 3 days. The fixed tissues were embedded in paraffin and sectioned into $8-\mu \mathrm{m}$ slices. The paraffinized slides were then deparaffinized twice with xylene and hydrated in $100 \%, 95 \%, 80 \%, 70 \%$, and $60 \%$ solutions of ethyl alcohol, in this sequence. It was assumed that HA and Cx-HA were excluded by this process.

The sectioned specimens were stained with hematoxylin and eosin (H\&E), SO, and an anti-TNF- $\alpha$ antibody. SO and TNF- $\alpha$ staining were performed by a method similar to the one described in the in previous work in references 18 and 19.

The SO- and TNF- $\alpha$-positive area was calculated as a percentage of the total cartilage area using ImageJ software (National Institutes of Health, Bethesda, MD, USA) in three randomly selected visual fields for each sample. Cartilage thickness was calculated using H\&E-stained samples in ImageJ from three randomly selected visual fields for each sample. 
(a)
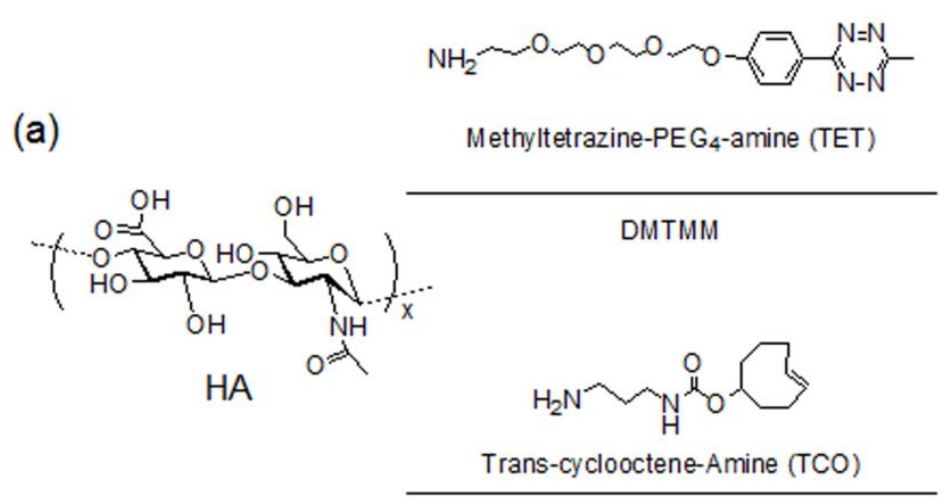

DMTMM

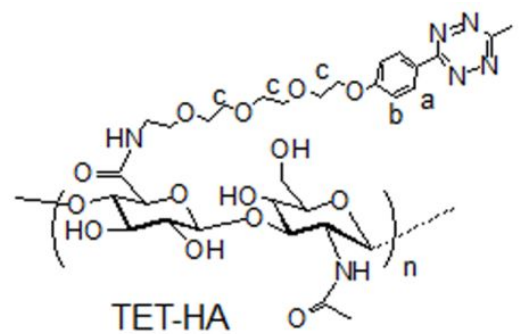

(b)
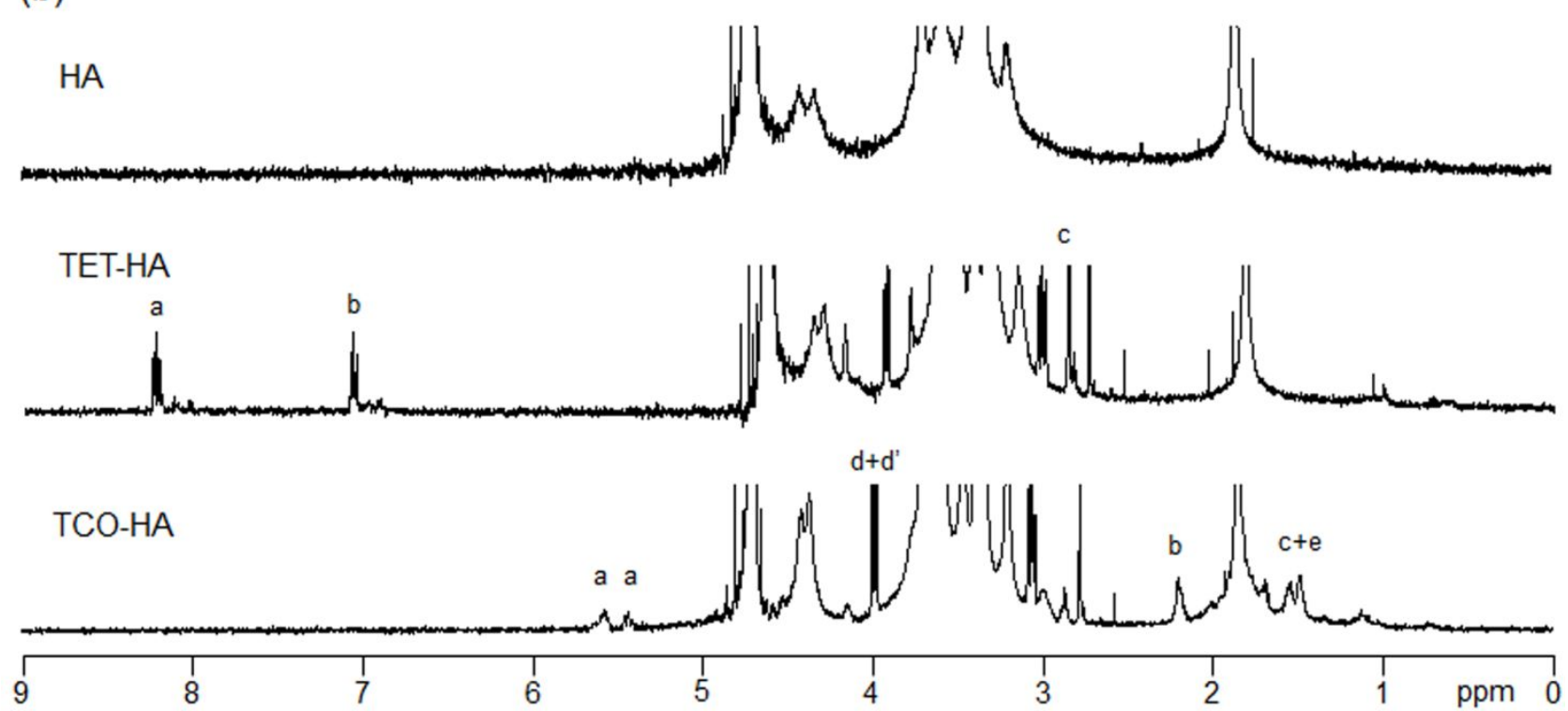

Figure S1. (a) Scheme of TET-HA and TCO-HA and (b) ${ }^{1} \mathrm{H}-\mathrm{NMR}$ of HA, TET-HA and TCO-HA. 

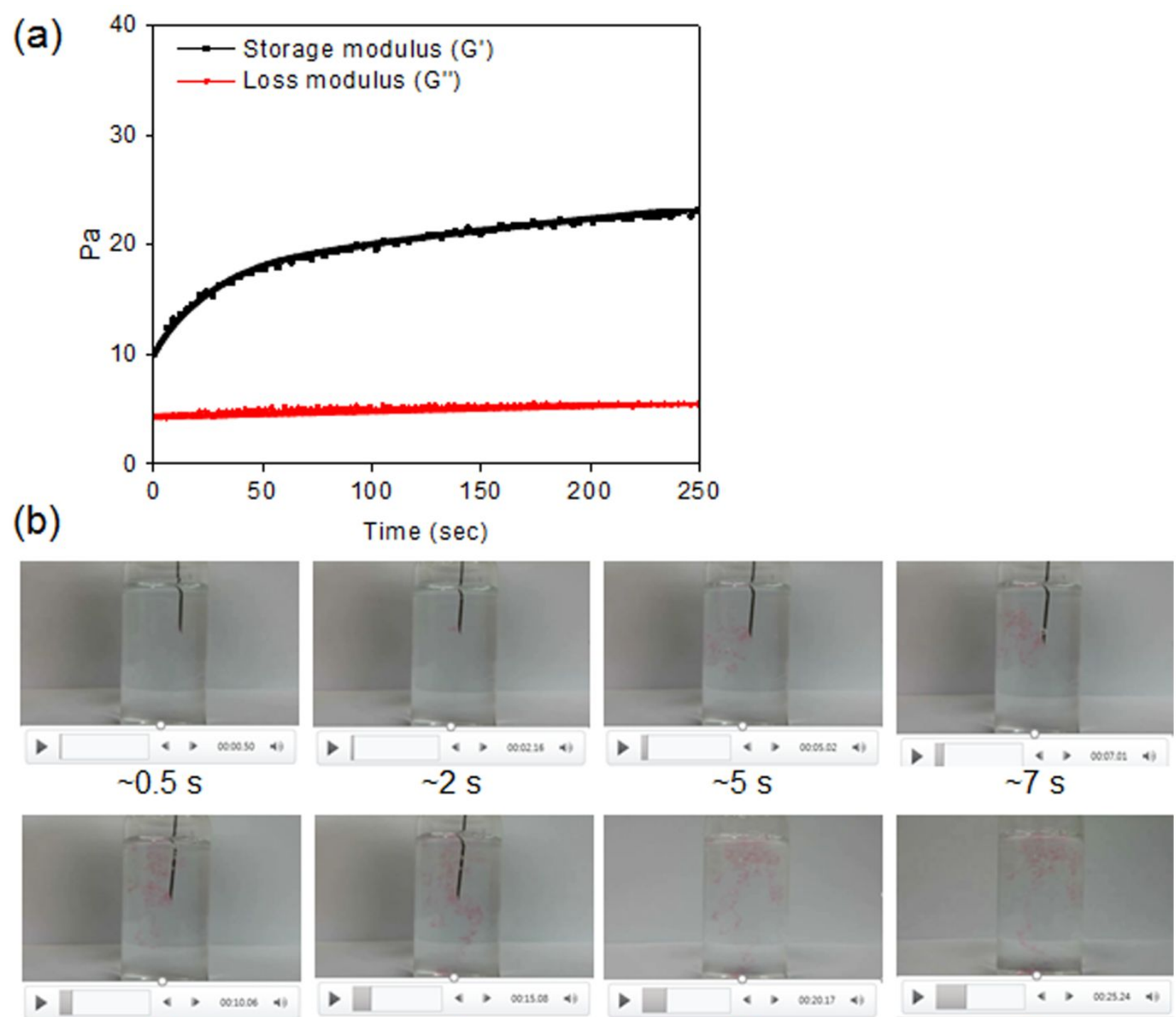

$\sim 10 \mathrm{~s}$

$\sim 15 \mathrm{~s}$

$\sim 20 \mathrm{~s}$
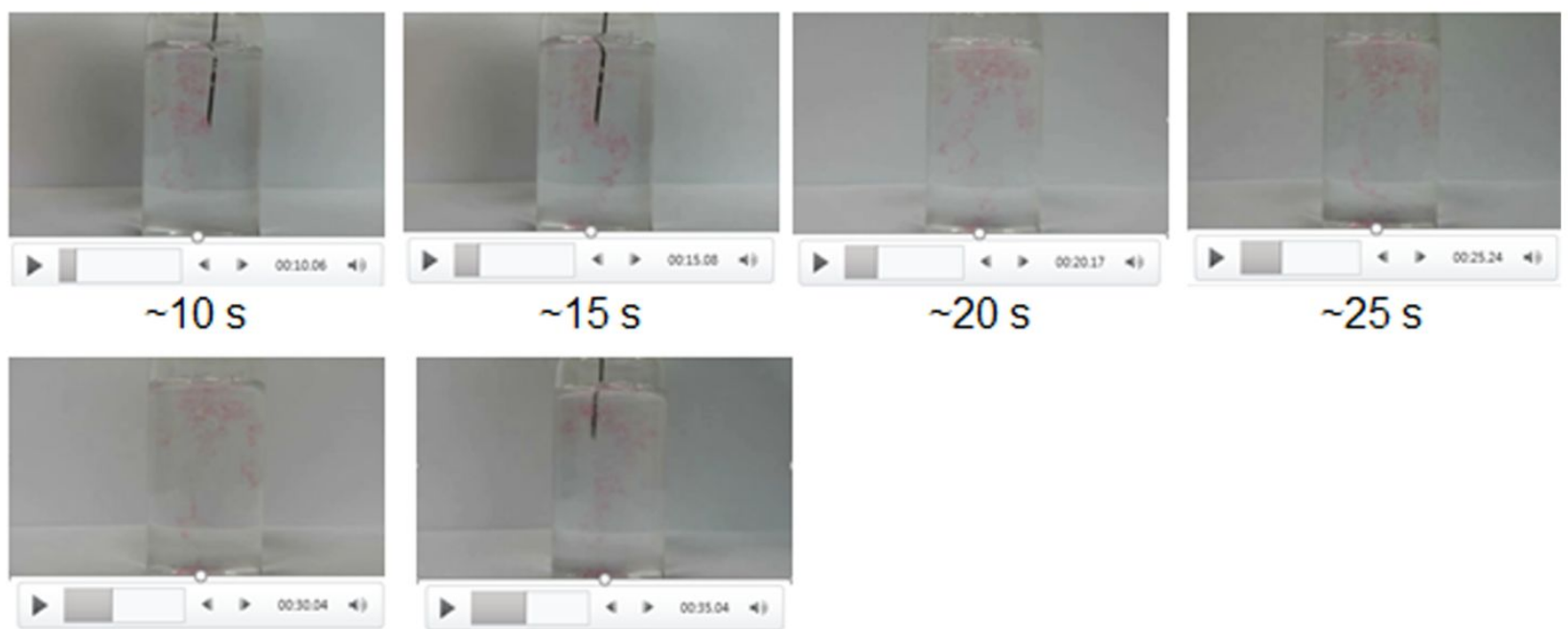

$\sim 30 \mathrm{~s}$

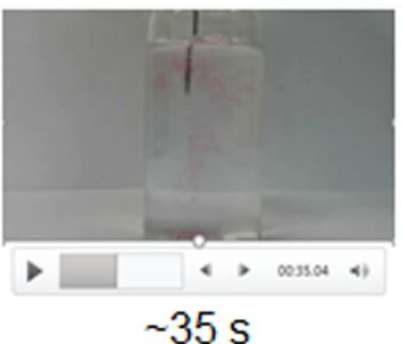

$\sim 25 \mathrm{~s}$

Figure S2. (a) Storage and loss moduli of Cx-HA hydrogels and (b) photo of ejecting TET-HA and TCO-HA through a 26 G syringe needle from each compartment of a dual-barrel syringe without clogging in PBS for $35 \mathrm{~s}$. 

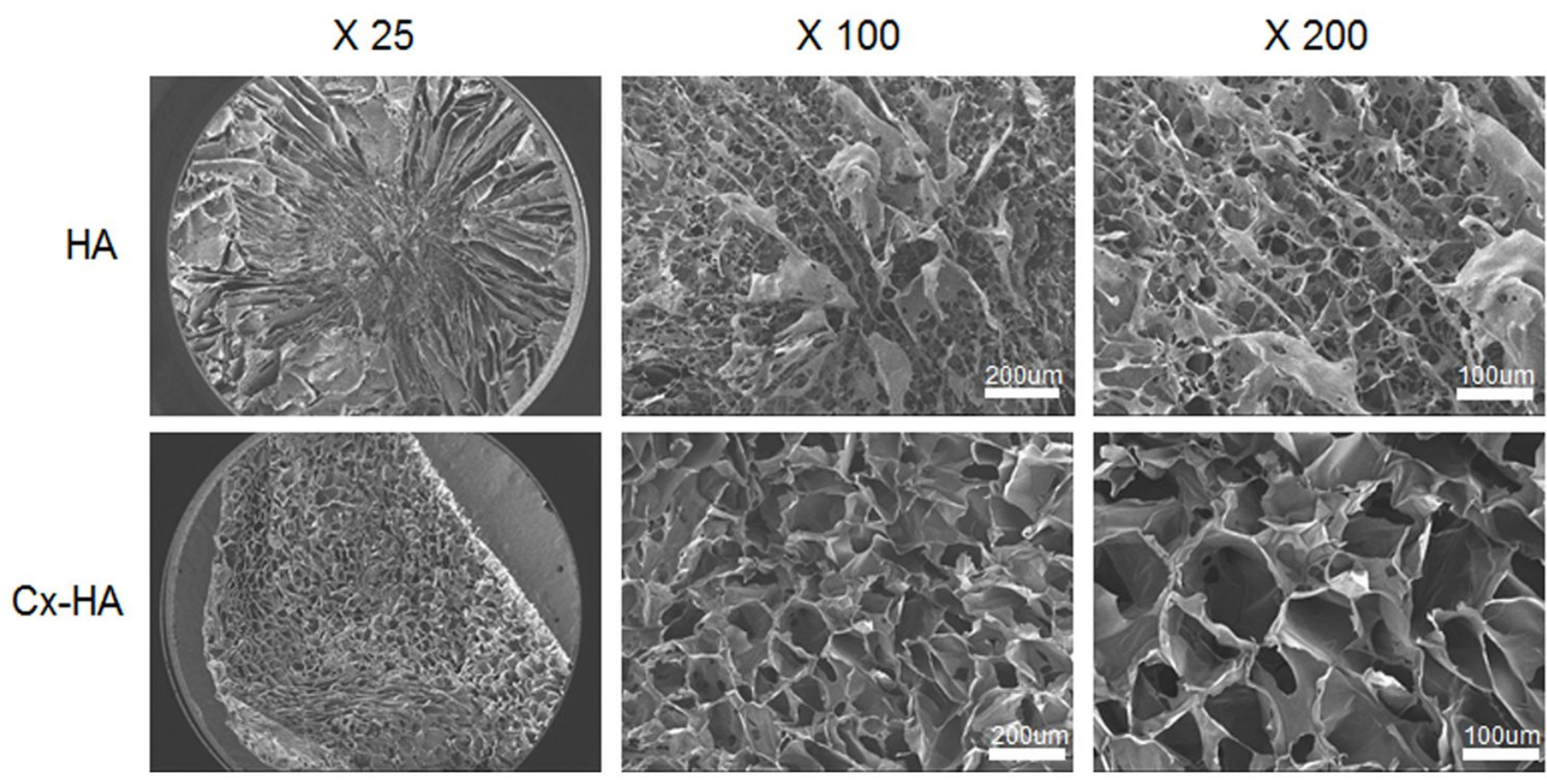

Figure S3. SEM of HA and Cx-HA hydrogel. 
(a)

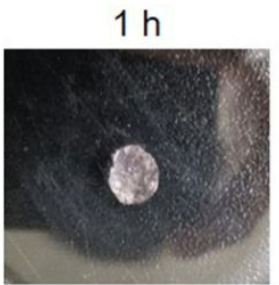

(b)
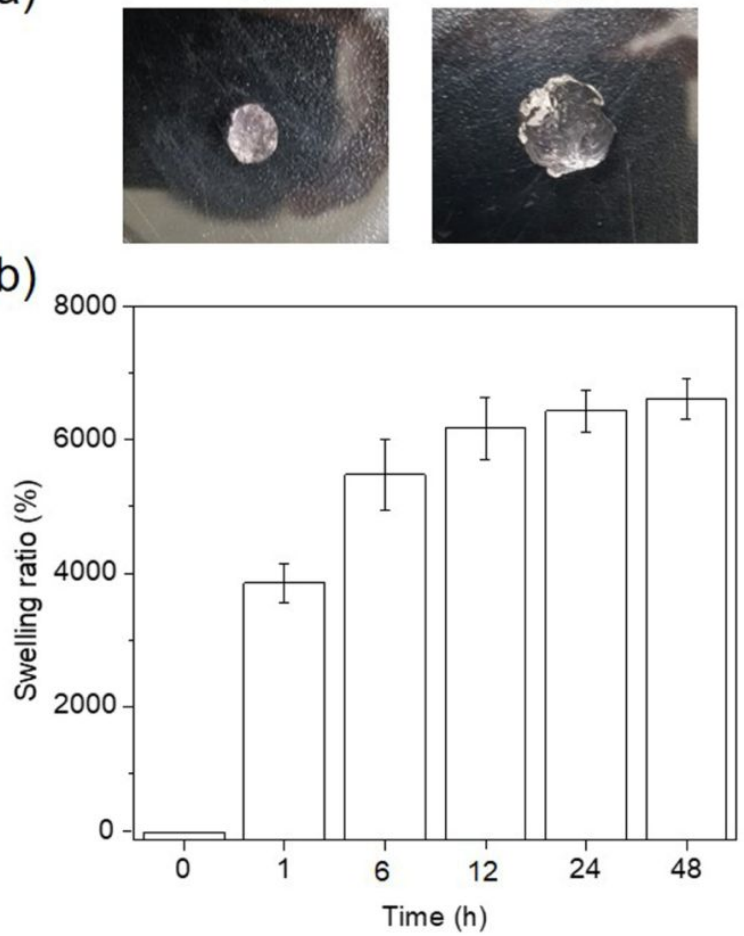

(c)

(d)

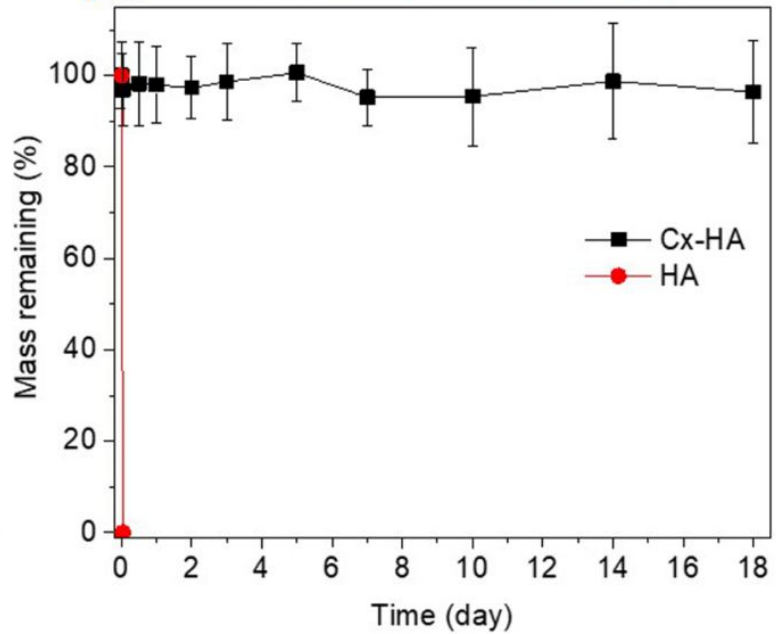

Figure S4. (a) Images of swelling of $\mathrm{Cx}-\mathrm{HA}$ at $1 \mathrm{~h}$ and $48 \mathrm{~h}$, (b) swelling ratio (\%) of $\mathrm{Cx}-\mathrm{HA}$ versus time, (c) images of $\mathrm{Cx}-\mathrm{HA}$ at 1 day and 14 days and (d) mass change ratio (\%) of $\mathrm{Cx}-\mathrm{HA}$ versus time. 
(a) No treatment
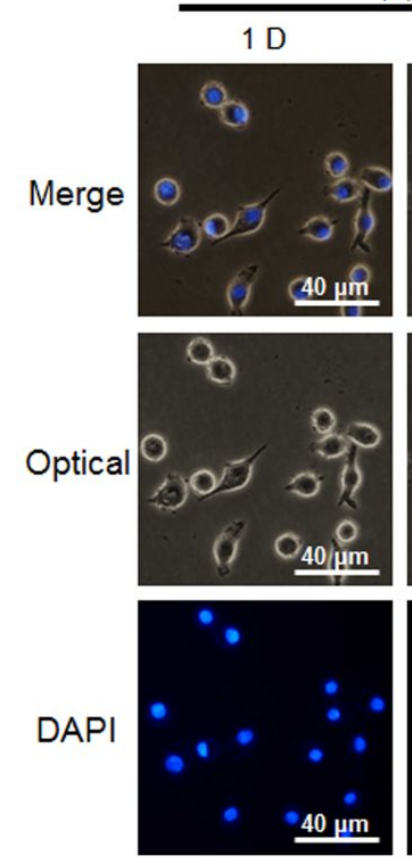

$4 \mathrm{D}$
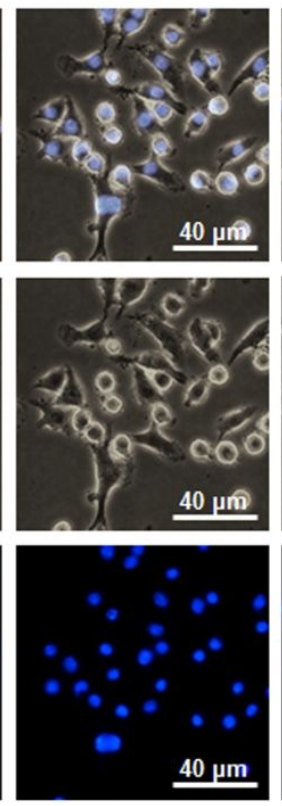
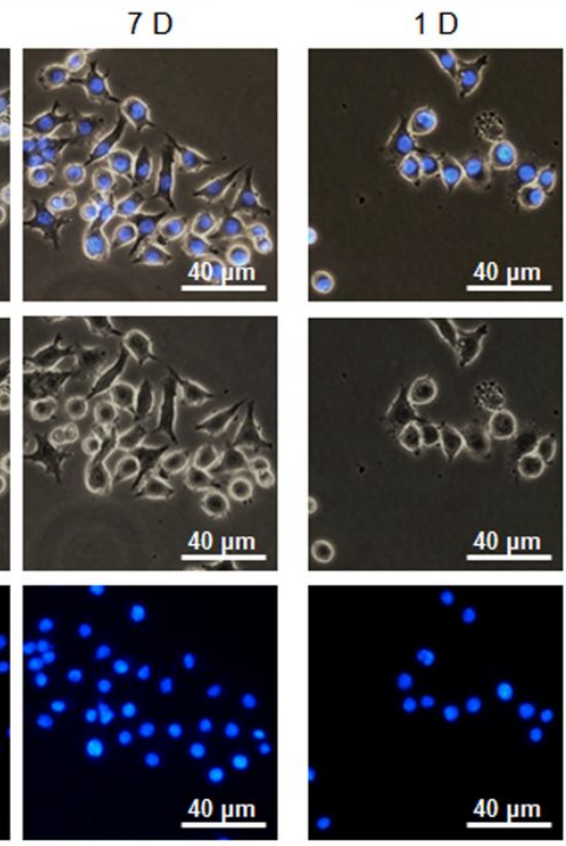

(b) MTX treatment
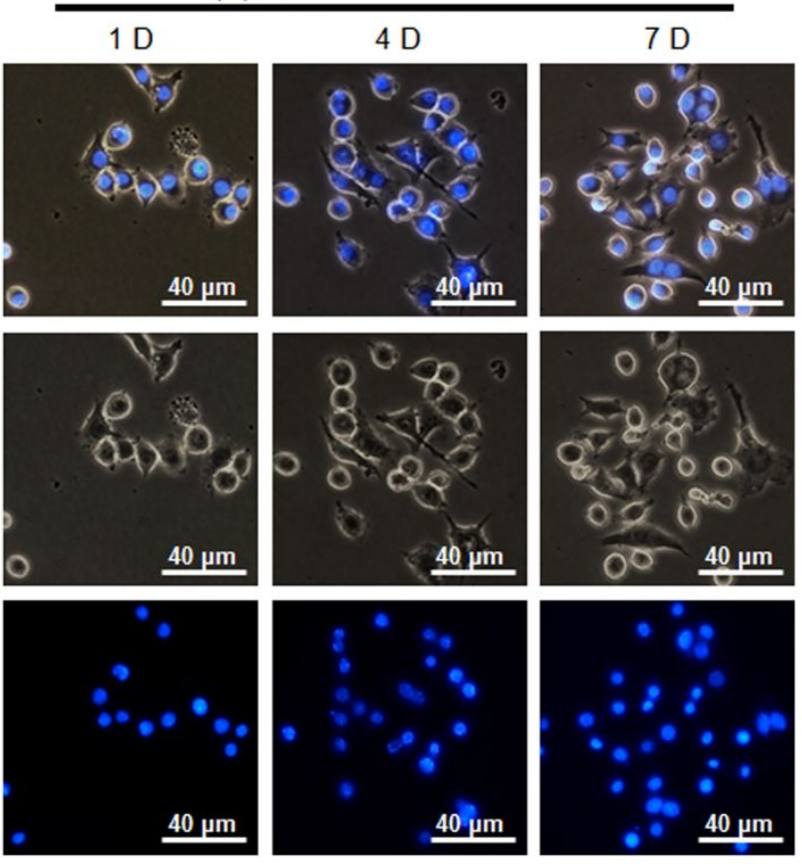

Figure S5. Confocal images of RAW 264.7 cells treated (a) without and (b) with MTX for one, four and seven days (magnification: $\times 400$, scale bar: $40 \mu \mathrm{m}$ ). 


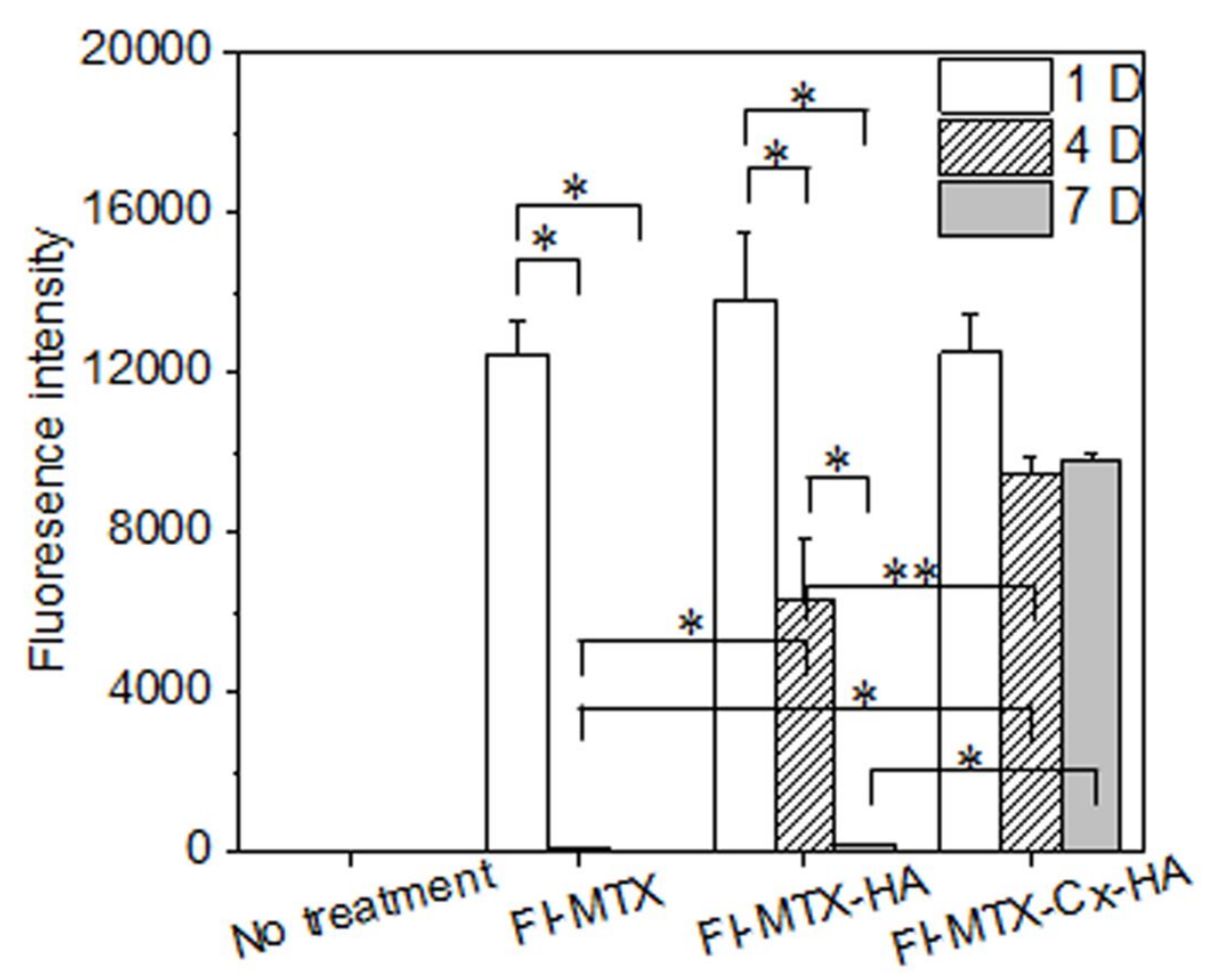

Figure S6. Fluorescence intensity of RAW 264.7 cells treated without a drug, with FI-MTX alone, FIMTX-HA, or FI-MTX-Cx-HA for 1, 4, and 7 days (determined from images of Figure 5$) \quad(* p<0.001$, $* * p<0.05)$. 


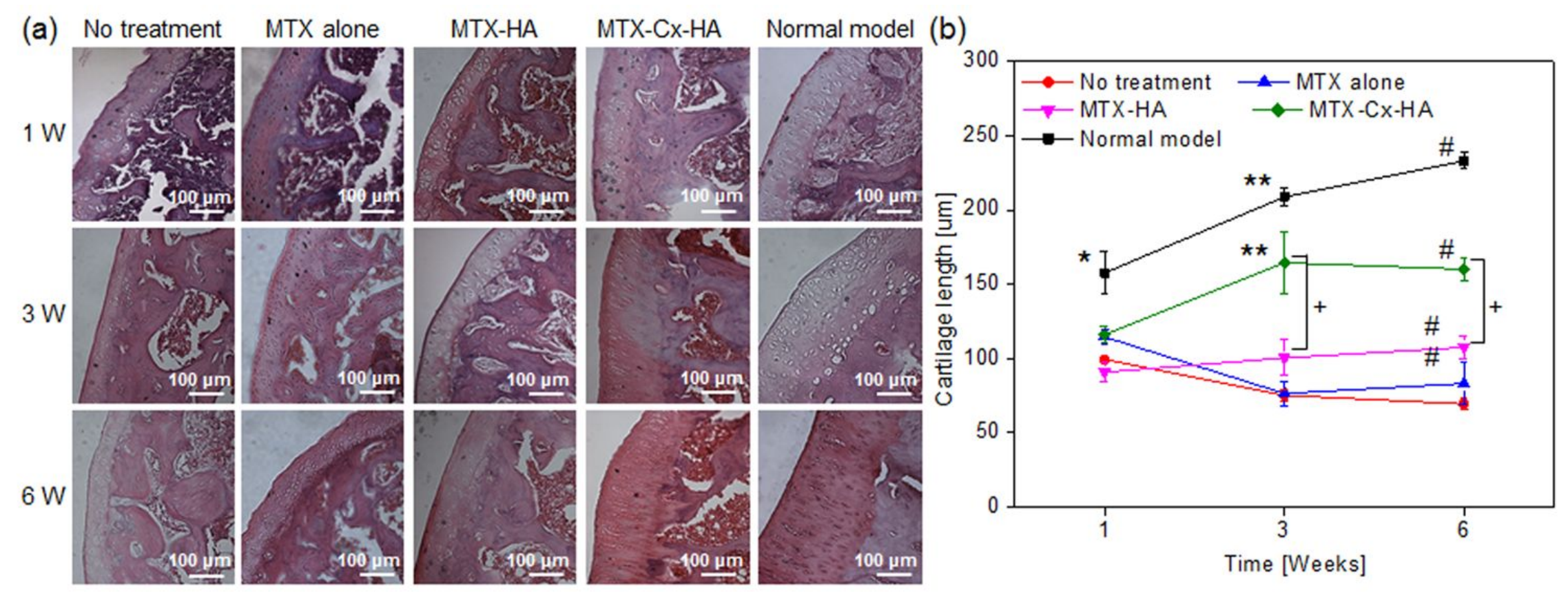

Figure S7. (a) H\&E staining and (b) the thickness of articular knee joint for RA rats received intra-articular injection without a drug, with MTX alone, MTX-HA, or MTX-Cx-HA as well as normal rats at 1, 3, and 6 weeks (magnification: $\times 200$, scale bars $=100 \mu \mathrm{m}) .(p<0.001$ versus non-treatment at $1\left[{ }^{*}\right], 3[* *]$, and $6[\#]$ weeks; $p<0.005$ versus no treatment at $6[\# \#]$ weeks; ${ }^{+} p<0.001$, MTX-CX-HA versus MTX-HA at 1,3, and 6 weeks). 

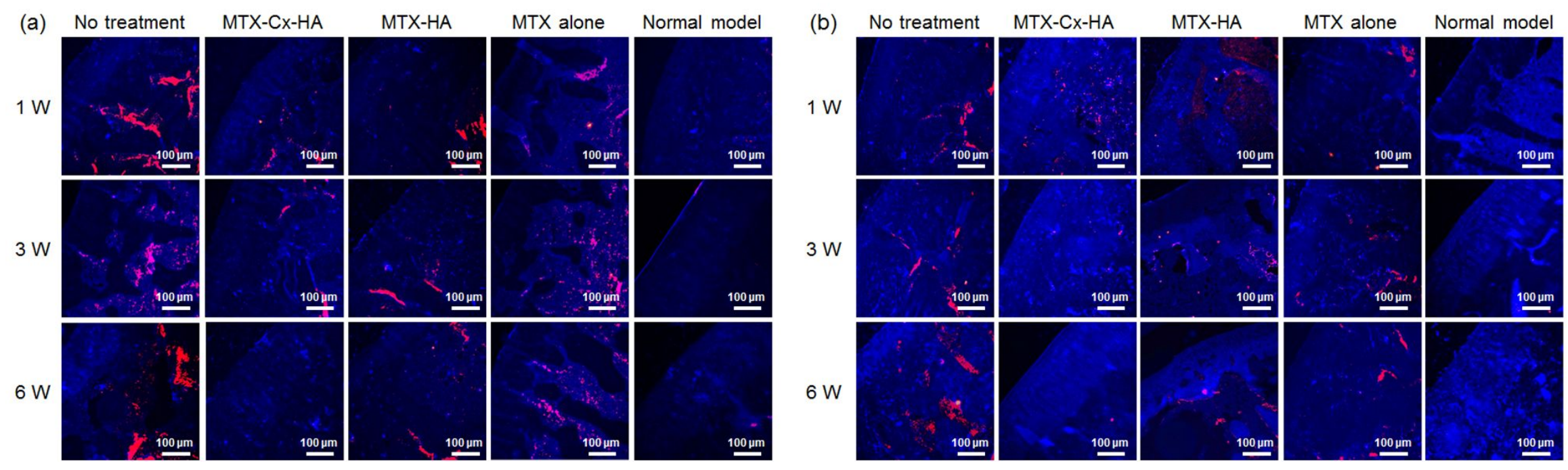

Figure S8. (a) CD4- and (b) ED1-staining of articular knee joint for RA rats received intra-articular injection without a drug (no treatment), with MTX alone, MTX-HA, or MTX-Cx-HA as well as normal rats at 1, 3, and 6 weeks (magnification: $\times 200$, scale bars $=100 \mu \mathrm{m}$ ). 


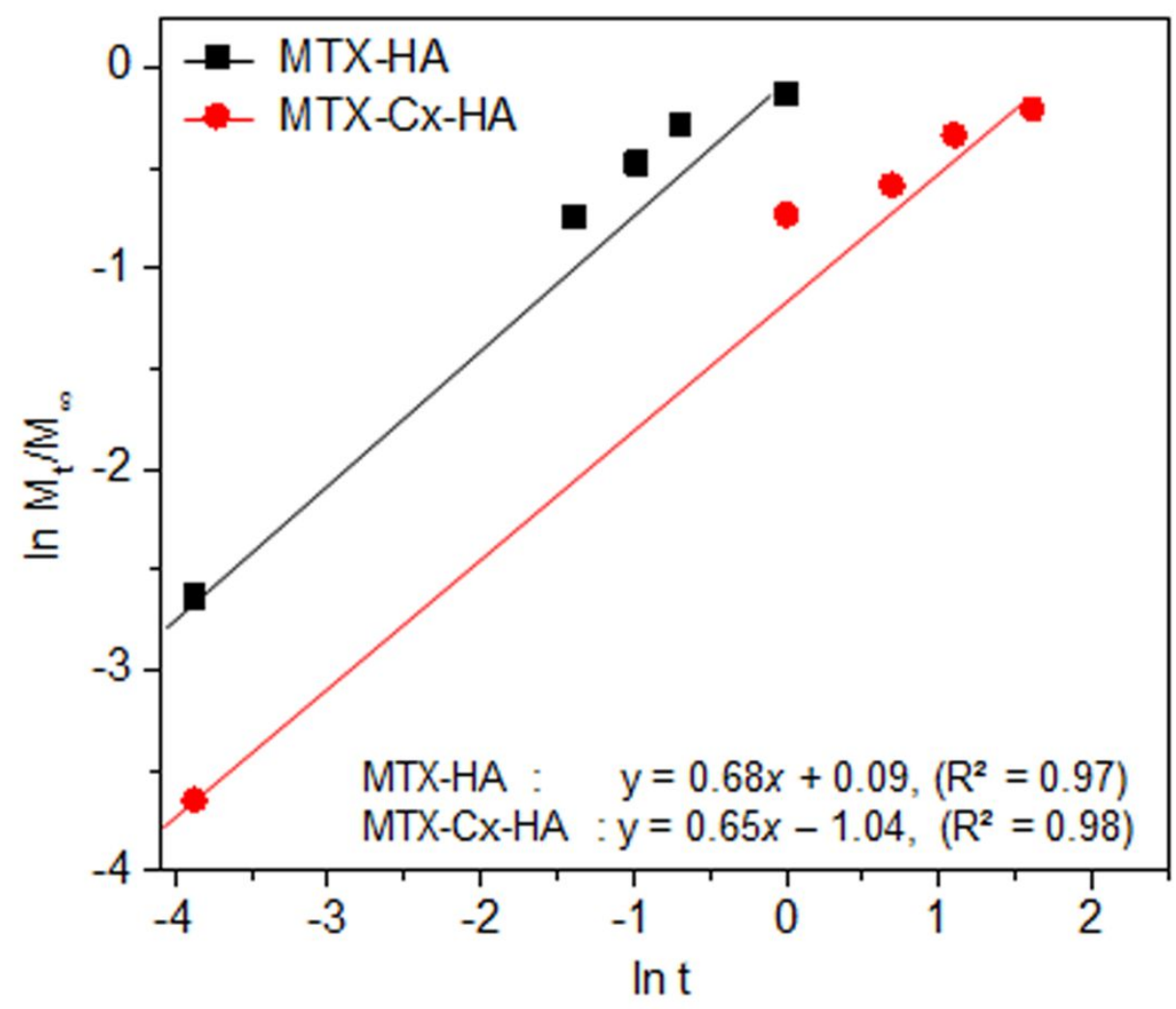

Figure S9. MTX released amount from HA or Cx-HA (described in Figure 3) versus release time.

*In accordance with one referee's suggestion, MTX release from HA or Cx-HA was compared to investigate a diffusion controlled manner or a degradation controlled manner as follow.

As in the case of swelling kinetics for the drug release kinetics, Korsmeyer-Peppas semi-empirical Equation was applied for the initial release stages ( $60 \%$ fractional release) [Korsmeyer, R.W.; Lustig, S.R.; Peppas, N.A. Solute and penetrant diffusion in swellable polymers. I. Mathematical modeling. J. Polym. Sci. Part B Polym. Phys. 1986, 24, 395-408.]:

$$
\frac{M_{\mathrm{t}}}{M_{\infty}}=k_{\mathrm{r}} t^{n_{\mathrm{r}}} \Rightarrow \ln \left(\frac{M_{\mathrm{t}}}{M_{\infty}}\right)=\ln k_{\mathrm{r}}+n_{\mathrm{r}} \ln t
$$

where $M_{\mathrm{t}} / \mathrm{M}_{\infty}$ is the fractional drug released, $M_{\mathrm{t}}$ and $M_{\infty}$ are the cumulative drug released amounts at 
time $\mathrm{t}$ and at equilibrium, respectively (or experimental maximum released amount taken at the plateau of the release curves), $k_{\mathrm{r}}$ is rate constant dependent on the characteristics of the drug loaded system and $\mathrm{n}_{\mathrm{r}}$ is the diffusional exponent which defines the type of the release mechanism.

A value of $n_{r} \sim 0.5$ is characteristic of the Fickian diffusion mechanism of the drug from the hydrogel. The values in interval $0.5<\mathrm{n}_{\mathrm{r}}<1$ are specific to an anomalous or non-Fickian behaviour. A case II of transport mechanism occurs when $\mathrm{n}_{\mathrm{r}}=1$, which means zero-order kinetics, while a special case II of transport mechanism is indicated by values $\mathrm{n}_{\mathrm{r}}>1$ [Korsmeyer-Peppas semi-empirical Equation]. The MTX release profiles are plotted as the cumulative percentage of MTX released versus time. The diffusion exponent in MTX release kinetics analysis of HA or MTX-Cx-HA depot was 0.68 and 0.65 value. This result implies that MTX release from HA or Cx-HA follows non-Fickian diffusion release mechanism. 